\title{
More and Better: Reappraisal Quality Partially Explains the Effect of Reappraisal Use on Changes in Positive and Negative Affect
}

Matthew W. Southward ${ }^{1}$, Anne C. Holmes ${ }^{2}$, Daniel R. Strunk ${ }^{2}$, \& Jennifer S. Cheavens ${ }^{2}$

\begin{abstract}
Author Note
Matthew W. Southward: https://orcid.org/0000-0002-5888-2769

Anne C. Holmes: https://orcid.org/0000-0002-4194-8012

Daniel R. Strunk: https://orcid.org/0000-0002-7444-0741

Jennifer S. Cheavens: https://orcid.org/0000-0002-3778-9346

Correspondence concerning this article should be addressed to Matthew W. Southward, Department of Psychology, University of Kentucky, 343 Waller Ave., Suite 303, Lexington, KY 40504; Phone: 859-562-1569; Email: southward@uky.edu

\section{Compliance with Ethical Standards}

\section{Disclosure of Potential Conflicts of Interest}

This work was partially supported by The Ohio State University Alumni Grants for Graduate Research and Scholarship (to Anne C. Holmes). The funding source had no involvement in the conduct or preparation of the research. All other authors declare no conflicts of interest with
\end{abstract} regard to the current study.

\section{Research Involving Human Participants and/or Animals}

All study procedures were approved by The Ohio State University's Institutional Review Board.

\section{Informed Consent}

Informed consent was obtained from all participants individually prior to their engagement in study procedures.

\section{In press at Cognitive Therapy and Research}

\footnotetext{
${ }^{1}$ Department of Psychology, University of Kentucky

${ }^{2}$ Department of Psychology, The Ohio State University
} 


\begin{abstract}
Background: A substantial body of research suggests that cognitive reappraisal is effective at improving momentary affect, but it remains unclear how reappraisal leads to these changes. We tested the quality of reappraisal as one potential mechanism.

Methods: A sample of 314 participants $\left(M_{\text {age }}=36.30 ; 51.0 \%\right.$ female; $69.4 \%$ White $)$ recruited online were instructed in the use of reappraisal and were asked to reappraise an upsetting memory for five minutes. Afterwards, participants rated the degree to which they used reappraisal during the task and independent raters coded the quality of participants' written descriptions. Participants also rated the intensity of positive and negative affect before and after the memory task.
\end{abstract}

Results: Reappraisal quality explained a significant proportion of the effect of reappraisal use on improvements in negative, $a b=-1.49, S E=.33,95 \% \mathrm{CI}[-2.17,-.90]$, and positive affect, $a b=$ $2.67, S E=.54,95 \%$ CI $[1.64,3.79]$. Depression symptom severity moderated these relations the indirect effects of reappraisal quality were stronger among those with fewer depressive symptoms.

Conclusions: These results suggest the quality with which reappraisal is used is one way through which reappraisal predicts improvements in affect, especially among people lower in depressive symptoms. Our findings enhance our understanding of the process of reappraisal and offer potential targets for interventions.

Keywords: reappraisal; quality; negative affect; positive affect; depression 


\section{More and Better: Reappraisal Quality Partially Explains the Effect of Reappraisal Use on Changes in Positive and Negative Affect}

Cognitive reappraisal is a skill in which people evaluate the accuracy, effectiveness, and/or meaning of their initial interpretations of a situation to influence their emotional experiences (Gross, 1998; Webb et al., 2012). Across 91 experimental studies, reappraisal demonstrated a medium-to-large sized effect on reducing immediate negative affect or increasing immediate positive affect $(d=.45$; Webb et al., 2012). Although people at higher levels of depression tend to use reappraisal less frequently than those at lower levels of depression $(r=-$ .17; Aldao et al., 2010), researchers have repeatedly shown that people with depression do not lack the ability to implement reappraisal (Liu \& Thompson, 2017; Yoon \& Rottenberg, 2019) and that reappraisal is similarly effective at changing momentary affect when used by people with or without depressive disorders (Gruber et al., 2014; Millgram et al., 2015; Quigley \& Dobson, 2014; Smoski et al., 2014). Despite this substantial evidence that reappraisal is generally effective in changing positive and negative affect, it remains unclear how reappraisal contributes to these affective changes. Identifying how reappraisal impacts changes in affect can inform how to target reappraisal to enhance the effectiveness of this skill.

Researchers have investigated several different potential paths through which reappraisal influences affective outcomes. For instance, researchers have investigated the number of reappraisals people generate (Barber \& DeRubeis, 1992; Rowlands et al., 2020), the speed with which people generate reappraisals (Rowlands et al., 2020), and the inventiveness or creativity of the reappraisals generated (Weber et al., 2014; Wu et al., 2017; 2019; Zeier et al., 2020). Of these potential mechanisms, only the ability to generate a greater number of reappraisals has been associated with better affective outcomes (Barber \& DeRubeis, 1992) when participants 
were not specifically trained beforehand.

One potentially influential link between the use of reappraisal and improvements in affect is the quality with which it is used. Quality can be defined as "how well a person uses a strategy in accordance with how it was conceptualized or taught, regardless of whether or not it leads to the intended outcome" (Southward et al., 2021). This definition is meant to distinguish how well a person uses reappraisal (i.e., quality) from how well reappraisal reduces negative affect (i.e., effectiveness) so as not to conflate the process of reappraisal with its downstream emotional outcomes. In response to the initial interpretation "I'm worthless", a lower quality reappraisal might be, "I'm not worthless." Although this new thought is less negative and contrasts with the original thought, there is no consideration of evidence for or against the initial thought and the reappraisal has not been elaborated. By contrast, a higher quality reappraisal might be, "Every person, including me, is inherently worthwhile. I'm also a committed partner who works hard at my job and cares for people in my community" (Southward et al., 2021).

One method researchers have used to study reappraisal quality is the Ways of Responding Scale (WOR; Barber \& DeRubeis, 1992). This measure presents participants with six hypothetical stressful scenarios followed by two to three initial negatively-valenced thoughts about each scenario. Participants then describe their further thoughts in each situation without additional prompting so as not to bias their descriptions. Independent raters use guidelines established by Barber and DeRubeis to rate the quality of each written response by considering how well the response addresses the initial thoughts or situation, how many different responses are generated, and how likely the responses as a whole are to reduce negative affect in a typical participant. Using the WOR, researchers have shown that reappraisal quality is related to, yet distinct from, frequency of strategy use (rs: .06-.44; Barber \& DeRubeis, 1992; Southward \& 
Cheavens, 2020), general self-control abilities ( $r$ s: .22-.46; Barber \& DeRubeis, 1992), and depressive symptoms (rs: -.31 - -.18; Barber \& DeRubeis, 1992; Yin et al., 2018) in community, clinical, and treatment-seeking samples.

There is reason to believe that reappraisal quality might be an important construct in understanding how reappraisal functions. As such, researchers have investigated whether reappraisal quality predicts affective responses. In one experimental study, previously depressed patients who had responded to cognitive therapy for depression completed a sad mood induction task one week after finishing treatment. Those patients who demonstrated greater reappraisal quality at the end of treatment also reported less depressive reactivity during the task (Strunk et al., 2013). Similarly, in a naturalistic study, undergraduate students reported their levels of depression before and after receiving exam grades three times throughout an academic term (Adler et al., 2013). The authors selected occasions when students received grades lower than their goal. Students who demonstrated higher reappraisal quality at the start of the semester reported less depressive symptom reactivity after receiving disappointing exam grades. This effect was significantly stronger among participants reporting higher levels of depressive symptoms, suggesting that how well people use reappraisal may vary by the severity of their depressive symptoms.

Together, these results suggest that the quality with which people use reappraisal in general can predict reappraisal success in specific stressful situations, as measured by lower depressive symptom reactivity. However, in both studies, reappraisal quality was measured using responses to hypothetical stressful situations days or months before the stressful situations. To more firmly establish whether and to what degree reappraisal quality explains the association between reappraisal use and affective change, it is necessary to assess both reappraisal quality 
and affective responses to the same stressor while a person's response to the stressor unfolds.

\section{Current Study}

In the current study, we examined the degree to which reappraisal quality explained the relation between reappraisal use and changes in negative affect in response to negative autobiographical memories. We hypothesized that (1) participants who used reappraisal to a greater degree would report greater decreases in negative affect. Further, we hypothesized that (2a) reappraisal quality would explain a significant amount of variability in the relation between reappraisal use and changes in negative affect. Given the small-to-medium sized relation between reappraisal quality and frequency, we hypothesized that (2b) the effect of reappraisal quality would remain even when adjusting for habitual reappraisal frequency. Finally, given the meta-analytic relation between reappraisal use and psychopathology, we explored (3) whether depressive symptoms moderated these effects.

\section{Methods \& Materials}

\section{Participants}

An initial sample of 408 potential participants was recruited through Amazon's Mechanical Turk (MTurk) service. To be eligible for the study, participants were required to be 18 years old or older, located in the United States, and to have achieved a $95 \%$ or higher performance rating on previous MTurk studies. Of the initial 408 potential participants, 81 (19.9\%) did not complete study procedures and 13 (3.2\%) did not comply with study procedures, as indicated by an absent or grossly incorrect text summary of a video presented at the beginning of the study. These exclusions resulted in 314 participants who were included in the final analyses, all of whom passed all attention check items.

Participants were 36.30 years old $(S D=11.39)$ on average, with a majority identifying as 
female $(n=160 ; 51.0 \%)$, White $(n=218 ; 69.4 \%)$, and non-Hispanic or Latino/a $(n=281$;

$89.5 \%)$. A plurality of participants had completed a four-year college degree $(n=112 ; 35.7 \%)$.

Excluded participants did not significantly differ from included participants on any demographic variables $(p s>.11)$.

\section{Measures}

\section{Demographics}

Participants first completed a demographics form. They self-reported their age, gender, racial and ethnic background, and highest level of education attained.

\section{State Emotion Regulation Inventory - Reappraisal Subscale}

The State Emotion Regulation Inventory - Reappraisal subscale (SERI-R; Katz et al., 2017) is a 4-item subscale designed to measure to the momentary use of reappraisal (e.g., "I tried to reevaluate the situation more positively"). Participants rate the extent to which they agree with each item on a scale from 1 (strongly disagree) to 7 (strongly agree). Items are averaged to create a total score, with higher scores indicating greater situational use of reappraisal. SERI-R items demonstrated excellent internal consistency $\left(\right.$ McDonald's omega $\left.[\omega]^{1}=.91\right)$.

\section{Reappraisal Quality}

To assess the quality of participants' reappraisals, participants provided written descriptions of how they reappraised a sad memory (see Reappraisal Instructions and Implementation section below for more details). Six undergraduate coders, unaware of participants' other responses and information, independently rated the quality of each reappraisal description from 1 (low quality) to 5 (high quality). Using Barber and DeRubeis' (1992)

\footnotetext{
${ }^{1}$ McDonald's omega is a more accurate and general expression of Cronbach's alpha because it allows individual items to exhibit different loadings on an underlying single factor (i.e., McDonald's omega does not assume essential tau-equivalence or unidimensionality but can be used to evaluate the plausibility of a single factor structure; Hayes $\&$ Coutts, 2020).
} 
guidelines, reappraisal quality was operationalized as how well the participant reframed the sad memory in a less negative way. Specifically, higher quality reappraisals included (a) more detail, (b) more unique perspectives on the memory, and (c) greater plausibility (e.g., if the sad memory is failing a class, reminding oneself that one class does not determine their future, rather than they will somehow erase this grade).

\section{Negative and Positive Affect}

To assess momentary affect, participants rated the extent to which they were currently feeling four emotions (i.e., fear/nervousness, disgust, sadness, and happiness) on a visual analogue scale from 0 (not at all) to 100 (extremely). We used a visual analogue scale to assess emotional intensity because this scale best approximates the theoretically continuous nature of emotional intensity and allows for greater variability in outcomes to better detect small effects (Krieg, 1999). Because people experience multiple negative emotions at the same time relatively

frequently (e.g., Watson \& Stanton, 2017), we calculated a composite score of negative affect by taking the mean of all three negatively-valenced emotions separately at each timepoint. Negative affect items demonstrated good internal consistency at baseline $(\omega=.82)$ but questionable internal consistency after the reappraisal implementation $(\omega=.68)$. Because positive affect may reflect a more unitary construct than negative affect (Jacobson et al., 2020; Watson \& Stanton, 2017), we only included one emotion item (happiness) to represent positive affect.

\section{Center for Epidemiologic Studies - Depression Scale}

The Center for Epidemiologic Studies - Depression Scale (CES-D; Radloff, 1977) is a 20-item measure of depressive symptoms over the previous week. Participants rate the frequency with which they have experienced each item from 0 (rarely or none of the time/less than 1 day) to 3 (most or all of the time/5-7 days). Items are summed to create a total score. CES-D items 
demonstrated excellent internal consistency in the present study $(\omega=.94)$.

\section{Emotion Regulation Questionnaire - Reappraisal Subscale}

The Emotion Regulation Questionnaire - Reappraisal subscale (ERQ-R; Gross \& John, 2003) is a 6-item subscale designed to assess participants' habitual use of reappraisal.

Participants rate the extent to which they agree with each item on a scale from 1 (strongly disagree) to 7 (strongly agree). Items are averaged to create a total score. ERQ-R items demonstrated excellent internal consistency $(\omega=.90)$.

\section{Procedure}

All study procedures were completed online via MTurk and Qualtrics (see Figure 1 for a visual depiction of the study flow and procedures). After selecting the study on MTurk, potential participants viewed an informed consent document and provided their consent to participate in the study. All participants then completed the demographics form and the ERQ-R. The present study was part of a larger investigation on the impact of implicit beliefs about emotions on emotional outcomes, so participants were randomized to view one of three brief videos: one communicating that emotions could be changed to induce a malleable mindset $(n=105)$, one communicating that emotions could not be changed to induce a fixed mindset $(n=97)$, and a mildly positive emotion-inducing video as a control condition $(n=112)$. This control condition was chosen because researchers have shown that mild, incidental positive emotion experiences do not influence reappraisal effectiveness (Yu et al., 2019).

\section{Negative Mood Induction}

Next, and in line with previously established procedures for inducing personally-relevant negative moods (Rusting \& Nolen-Hoeksema, 1998), participants received the following prompt: “Think of a time in your life when you felt upset or sad. Picture in your 'mind's eye' the 
surroundings as clearly as possible. See the people or objects; hear the sounds; experience the events happening to you. Write down a very brief (1 sentence) description or "title" of the memory below so that you know which memory you selected." After writing this one sentence description of the memory, participants rated the intensity of their positive and negative affect.

\section{Reappraisal Instructions and Implementation}

Participants were then presented with a brief passage describing cognitive reappraisal: "Recalling upsetting memories can bring up difficult and unpleasant thoughts and emotions. So, we'd like to give you a tool that may help you deal with the thoughts and emotions that this memory may bring up. This tool is called 'cognitive reappraisal' and it involves reframing the situation by thinking about it in a different way that reduces its negative meaning. As you go through the next task, try to reframe the memory and think about it in a different way." These instructions were kept intentionally brief and relatively non-specific to preserve individual differences in reappraisal use and quality during the following implementation task.

We then asked participants to spend five minutes reappraising the autobiographical memory they selected earlier. Participants were instructed: "Picture the event happening to you all over again. As you're re-imagining the event, try to think about the situation in a different way that makes you feel less negative. Use the space below to describe the memory in vivid detail. Write about what is happening, what you are thinking, and how you are feeling." Immediately after the five-minute implementation period, participants again reported the intensity of their positive and negative affect as well as the degree to which they used reappraisal during the implementation period. Finally, participants were thanked, debriefed, and paid \$2.00 for their participation). All study procedures were approved by the local Institutional Review Board. 


\section{Data Analytic Method}

We trained six undergraduate coders to independently rate the quality of participants' reappraisals. Coders met weekly with the second author (A.C.H.) to receive training in making these ratings. Each week, coders rated the quality of an independent set of reappraisal descriptions until they demonstrated acceptable inter-rater reliability for two consecutive weeks. We used Krippendorff's alpha to assess inter-rater reliability using the KALPHA macro (Hayes \& Krippendorff, 2007) with 5,000 bootstraps. Krippendorff's alpha ranges from $-1.00-1.00$ and quantifies the degree of agreement beyond chance between $n$ raters on interval or ratio levels of data with any degree of missingness. Krippendorff (2004) has suggested $\alpha$ s from .67-.80 indicate tentative reliability, and $\alpha \mathrm{s}>.80$ indicate acceptable reliability. Coders were required to demonstrate $\alpha s \geq .70$ for two consecutive weeks before rating study data. Coders were then assigned to rate a random subset of reappraisal descriptions such that each description was rated by three coders. Because coders demonstrated acceptable inter-rater reliability using the study data, Krippendorff's $\alpha=.79,95 \%$ CI [.76, .83], we averaged the ratings of all three coders for each description to calculate participants' reappraisal quality score.

We first examined descriptive statistics and bivariate correlations among our variables of interest. To test Hypothesis 1, that reappraisal use would predict change in affect during reappraisal implementation, we regressed negative affect after reappraisal implementation on negative affect after the mood induction and SERI-R scores. ${ }^{2}$ We repeated this analysis entering positive affect after reappraisal implementation and mood induction, respectively.

\footnotetext{
${ }^{2}$ Because experiences of single negative emotions are roughly as common as blends of multiple negative emotions (Watson \& Stanton, 2017) and multiple researchers have found that reappraisal may be differentially efficacious for different emotions (Demaree et al., 2006; Olatunji et al., 2017; Pasupathi et al., 2017; Southward et al., 2019), we reran our analyses with each specific negative emotion as an outcome (Tables S1-S5, Supplemental Materials).
} 
To test Hypothesis 2a, that reappraisal quality would explain significant variability in these relations, we conducted a mediation analysis using Model 4 of the PROCESS macro Version 3.4 (Hayes, 2017) with 10,000 percentile bootstraps. Although the limitations of mediation analyses for establishing causal effects are becoming more widely acknowledged (MacKinnon et al., 2000; Yzerbyt et al., 2018), mediation analyses allow for the decomposition of total effects into constituent parts. Specifically, and in the current study, the total effect of reappraisal use on affective changes (the $c$ path; Figure 2) can be decomposed into the direct effect of reappraisal use (the c' path; Figure 2) and the indirect effect through reappraisal quality (the $a b$ path; Figure 2) because, by definition, $c=a b+c$ ' (Yzerbyt et al., 2018). This model allows us to examine what proportion of the total effect of reappraisal use is explained by the indirect effect of reappraisal quality. ${ }^{3}$ We entered SERI-R as the focal predictor, reappraisal quality as the mediator, post-implementation negative (or positive) affect as the outcome, with post-mood induction negative (or positive) affect as a covariate. To test Hypothesis $2 \mathrm{~b}$, that the indirect effect would hold after adjusting for habitual reappraisal frequency, we re-ran the model from Hypothesis 2a but included reappraisal frequency as a covariate.

Finally, to explore if depressive symptoms moderated these relations (Hypothesis 3), we included depressive symptoms as a moderator of all legs of the mediation model from Hypothesis $2 \mathrm{~b}$ using Model 59 of the PROCESS macro. We probed significant moderation effects using the Johnson-Neyman technique. Because participants were randomly assigned to receive an experimental mindset induction as part of the larger study, we included experimental

\footnotetext{
${ }^{3}$ We note that the (lack of) a temporal relation between reappraisal use and reappraisal quality (the $a$ path) may more accurately be represented by the covariance between these variables. However, given the larger literature on interpreting mediation analyses and the relative ease of interpreting products of regression coefficients compared to products of regression coefficients and covariances, we have opted to conduct mediation analyses and explicitly note when these analyses do and do not imply causal and/or temporal relations.
} 
condition as a covariate in all analyses. Similarly, we included the number of words participants wrote in the implementation task as a covariate in all analyses to rule out word count as an alternative explanation for any effects of reappraisal quality. All variables except positive and negative affect were mean-centered prior to analysis to ease interpretation, and all analyses were conducted in SPSS Version 26 (IBM Corp., 2019). All code is available at https://osf.io/ruqsf/?view_only=f560bf4267564f6e8890a754c8580894.

\section{Results}

\section{Descriptive Statistics and Correlations Among Variables of Interest}

Demographic characteristics were generally unrelated to reappraisal, affect, and depression symptom variables. The only two exceptions were participants who identified as nonWhite demonstrated lower reappraisal quality than those who identified as White, $r=-.12, p=$ .04 , and older participants reported fewer depressive symptoms than younger participants, $r=-$ $.16, p<.01$. Both of these relations were small in magnitude. Of note, 89 participants $(28.3 \%)$ scored at or above the threshold of 20 on the CES-D, which is indicative of clinically significant depressive symptoms (Vilagut et al., 2016).

Greater momentary reappraisal use (SERI-R) was significantly associated with greater reappraisal quality, $r=.56, p<.01$, and habitual reappraisal frequency (ERQ-R), $r=.14, p=.02$. However, reappraisal quality was not significantly associated with habitual reappraisal frequency (ERQ-R), $r=-.01, p=.93$. Greater momentary reappraisal use (SERI-R), $r=-.38, p<.01$, and reappraisal quality, $r=-.42, p<.01$, but not habitual reappraisal frequency (ERQ-R), $r=-.07, p$ $=.24$, were related to lower post-implementation negative affect. Greater momentary reappraisal use (SERI-R), quality, and habitual frequency (ERQ-R) were all significantly related to higher post-implementation positive affect, $r s: .20-.43, p s<.01$. Only habitual reappraisal frequency 
(ERQ-R) was significantly related to lower past-week depressive symptoms, $r=-.30, p<.01$; momentary reappraisal use (SERI-R) and quality were not significantly related to past-week depressive symptoms, $r \mathrm{~s}:-.09--.06, p \mathrm{~s}>.10$.

\section{Reappraisal Use Predicting Affect Change}

Although negative affect did not change significantly from mood induction to reappraisal implementation, $t(312)=1.29, p=.20,95 \% \mathrm{CI}[-.57,2.96], d=.07,129$ participants $(41.1 \%)$ reported a numeric decrease in negative affect $(M=-10.81, S D=11.76$, range $=-66.67--.33)$. Consistent with Hypothesis 1, participants who used reappraisal to a greater degree during the implementation period (SERI-R) reported a greater residualized decrease in negative affect than those who used reappraisal to a lesser degree, $B=-4.15, S E=.52, p<.01,95 \%$ CI $[-5.16$, 3.13]. Similarly, although positive affect did not change significantly from mood induction to reappraisal implementation, $t(312)=-.35, p=.72,95 \% \mathrm{CI}[-3.04,2.07], d=-.02,132$ participants $(42.0 \%)$ reported a numeric increase in positive affect $(M=17.57, S D=17.92$, range $=1.00-91.00)$. Also consistent with Hypothesis 1, participants who used reappraisal to a greater degree (SERI-R) reported a greater residualized increase in positive affect than those who used it to a lesser degree, $B=6.15, S E=.77, p<.01,95 \% \mathrm{CI}[4.65,7.66]$.

\section{The Indirect Effect of Reappraisal Use on Affect Change Through Reappraisal Quality}

Consistent with Hypothesis 2a, there was a significant indirect effect between reappraisal use (SERI-R) and negative affect change through reappraisal quality, $a b=-1.49, S E=.33,95 \%$ CI $[-2.17,-.90]$ (Figure 2A). Specifically, people who reported using reappraisal to a greater degree (SERI-R) tended to demonstrate higher reappraisal quality, $a=.41, S E=.04, p<.01$, $95 \%$ CI $[.34, .48]$, and those who demonstrated higher reappraisal quality reported a greater decrease in negative affect across the implementation period, $b=-3.66, S E=.81, p<.01,95 \%$ 
CI $[-5.25,-2.07]$. This indirect effect represented $35.0 \%$ of the total effect of reappraisal use on changes in negative affect.

Similarly, and also consistent with Hypothesis 2a, there was a significant indirect effect between reappraisal use (SERI-R) and positive affect change through reappraisal quality, $a b=$ 2.67, $S E=.54,95 \%$ CI $[1.64,3.79]$ (Figure 2B). People who reported using reappraisal to a greater degree (SERI-R) demonstrated higher reappraisal quality, $a=.42, S E=.04, p<.01,95 \%$ CI $[.35, .49]$, and those who demonstrated higher reappraisal quality reported a greater increase in positive affect across the implementation period, $b=6.38, S E=1.15, p<.01,95 \% \mathrm{CI}[4.12$, 8.64]. This indirect effect represented $42.6 \%$ of the total effect of reappraisal use on changes in positive affect.

Consistent with Hypothesis $2 \mathrm{~b}$, when including habitual reappraisal frequency (ERQ-R) as a covariate in these models, the indirect effects of reappraisal quality remained largely unchanged. There was a significant indirect effect between reappraisal use (SERI-R) and negative affect change through reappraisal quality, $a b=-1.49, S E=.33,95 \%$ CI $[-2.18,-.88]$, representing $34.2 \%$ of the total effect of reappraisal use (SERI-R) on reductions in negative affect. There was also a significant indirect effect between reappraisal use (SERI-R) and positive affect change through reappraisal quality, $a b=2.71, S E=.56,95 \%$ CI $[1.67,3.88]$, representing $42.9 \%$ of the total effect of reappraisal use.

\section{Do Depressive Symptoms Moderate the Effects of Reappraisal?}

When exploring Hypothesis 3, depressive symptoms significantly moderated the indirect effect of reappraisal quality on the relation between reappraisal use (SERI-R) and change in negative affect, index of moderated mediation: .06, $S E=.03,95 \%$ CI $[.01, .11] .{ }^{4}$ Depressive

\footnotetext{
${ }^{4}$ PROCESS only calculates an index of moderated mediation for models with a moderator of one leg of a mediational path. Thus, after we reviewed the results of Model 59, we used Model 14 to calculate the index of
} 
symptoms did not moderate the relations between reappraisal use (SERI-R) and quality, $B=-$ $.0004, S E=.003, p=.90,95 \% \mathrm{CI}[-.006, .006]$, or between reappraisal use (SERI-R) and residualized change in negative affect, $B=-.01, S E=.05, p=.85,95 \%$ CI $[-.11, .09]$. However, depressive symptoms did moderate the relation between reappraisal quality and residualized change in negative affect, $B=.15, S E=.07, p=.02,95 \%$ CI $[.02, .29]$. Probing this effect with the Johnson-Neyman technique, we found that, among people generally below the suggested clinical threshold of depressive symptoms (CES-D $<22.22$; zCES-D $<.70 ; 78.1 \%$ of the sample), those who demonstrated greater reappraisal quality reported a greater decrease in negative affect ( $p$ s $<.05 ;$ Figure 3A). Among those generally above the suggested clinical threshold (CES-D $\geq$ $22.22 ; z$ CES-D $\geq .70 ; 21.9 \%$ of the sample), reappraisal quality was unrelated to changes in negative affect $(p s>.05)$. Thus, although the direct effect of reappraisal use (SERI-R) on changes in negative affect was relatively consistent regardless of participants' depressive symptoms, the indirect effect of reappraisal quality was weaker among people with more depressive symptoms (Table 2).

Similarly, when exploring Hypothesis 3, depressive symptoms moderated the indirect effect of reappraisal quality on the relation between reappraisal use (SERI-R) and change in positive affect, index of moderated mediation: $-.08, S E=.03,95 \%$ CI $[-.14,-.02] .{ }^{5}$ Depressive symptoms did not moderate the relations between reappraisal use (SERI-R) and quality, $B=-$ $.0008, S E=.003, p=.81,95 \% \mathrm{CI}[-.007, .005]$, or between reappraisal use (SERI-R) and residualized change in positive affect, $B=.06, S E=.07, p=.45,95 \% \mathrm{CI}[-.09, .20]$. However, depressive symptoms did moderate the relation between reappraisal quality and residualized

moderated mediation. Model 14 was selected because, in this model, the moderator only moderates the $b$ path (i.e., reappraisal quality to change in negative affect).

${ }^{5}$ This index of moderated mediation is also based on Model 14 in PROCESS, as we used with the negative affect results. 
change in positive affect, $B=-.22, S E=.10, p=.02,95 \% \mathrm{CI}[-.41,-.03]$. Probing this effect with the Johnson-Neyman technique, we found that, among people generally below the clinical threshold for depressive symptoms (CES-D < 25.49; zCES-D $<.98 ; 83.2 \%$ of the sample), those who demonstrated greater reappraisal quality reported a greater increase in positive affect ( $p$ s < .05 ; Figure 3B). Among those generally above the clinical threshold for depressive symptoms (CES-D $\geq 25.49 ; z$ CES-D $\geq .98 ; 16.8 \%$ of the sample), reappraisal quality was unrelated to changes in positive affect $(p s>.05)$. Again, although the direct effect of reappraisal use (SERI-R) on positive affect was relatively consistent regardless of participants' depressive symptoms, the indirect effect of reappraisal quality was weaker among people with more depressive symptoms (Table 2).

\section{Discussion}

In this study, we found that people who used cognitive reappraisal to a greater degree when recalling an upsetting memory reported a greater decrease in negative affect and a greater increase in positive affect, consistent with our first hypothesis. These effects were partially explained by the quality with which participants used reappraisal, regardless of how frequently participants tended to use reappraisal in their lives, consistent with our second hypothesis.

Finally, we found that depressive symptoms moderated the indirect effects of reappraisal quality, such that the indirect effects of reappraisal quality were stronger among people reporting fewer depressive symptoms. Together, these results contribute to our understanding of how reappraisal functions and for whom it is most efficacious.

Consistent with previous work (Katz et al., 2017; McRae et al., 2012; Webb et al., 2012), people who used reappraisal to a greater degree reported greater decreases in negative affect and greater increases in positive affect during the mood induction than those who used reappraisal to 
a lesser degree. Further, as reported in supplemental materials, reappraisal use predicted decreases in sadness, fear, and disgust individually. These results provide further evidence that reappraisal is an effective emotion regulation strategy across a range of distressing emotions.

When testing our mediational hypothesis, we found that reappraisal quality explained roughly $30-40 \%$ of the total effect of reappraisal use on changes in negative and positive affect, even when accounting for participants' habitual frequency of reappraisal. This novel finding is the first to demonstrate that the quality with which reappraisal is used is a way in which reappraisal influences affective changes. It suggests that it is not just the act of reflecting on one's thoughts and considering alternative explanations that contributes to improvements in positive and negative affect, but that how well that process is enacted is also important.

Momentary reappraisal use was strongly associated with reappraisal quality, suggesting that the degree to which a person uses reappraisal in a given situation is indicative of, but not redundant with, how well they use the skill. Thus, reappraisal use and quality may represent two related but distinct paths contributing to affective change.

By contrast, habitual reappraisal use was unrelated to reappraisal quality. This result suggests that people who use reappraisal well do not necessarily have to use it very frequently, perhaps because these people tend to have lower levels of psychopathology in general and thus fewer negative emotional experiences to reappraise or these people experience a greater impact from relatively fewer, higher quality reappraisals than several lower quality reappraisal.

Developmentally, the lack of a relation between habitual reappraisal frequency and reappraisal quality may suggest that people do not learn to use higher quality reappraisals with repeated practice in everyday life. This result contrasts somewhat with previous estimates, in which habitual reappraisal use demonstrated small-to-medium sized associations with reappraisal 
quality (Barber \& DeRubeis, 1992; Southward \& Cheavens, 2020). The discrepancy between these results may reflect the content reappraised. In the current study, participants were asked to consider a single personal memory, whereas previous researchers have presented participants with several standardized hypothetical situations. Repeated responses to a range of hypothetical stressful situations may be more similar to the situations considered when participants make ratings of habitual reappraisal use. However, we encourage future researchers to explore the etiological determinants of reappraisal use and quality as these pathways may inform who benefits most from each dimension of reappraisal.

Quality did not explain the majority of variability in the effect of reappraisal use, which may indicate that other aspects of reappraisal also contribute to its effectiveness. These aspects may include a person's emotion regulation goals in the moment (Tamir et al., 2019), the specific tactic people use to reappraise a situation (McRae et al., 2012), the fit between the reappraisal tactic used and the emotion experienced (Vishkin et al., 2020), or even the degree to which a person believes in the reappraisal(s) they generate. We encourage future researchers to holistically test and compare the strength of these potential mechanisms to more comprehensively characterize the processes involved in the use of reappraisal.

Consistent with our exploratory hypothesis, depressive symptoms moderated the indirect effect of reappraisal quality. Specifically, the indirect effect of reappraisal quality on affective changes was strongest among those lower in depressive symptoms but non-significant among people with clinical levels of depressive symptoms. One possible explanation for this finding is that, among people with clinical levels of depression, the ability to focus one's efforts on the use of reappraisal to the exclusion of more maladaptive responses may be predictive of affective improvement regardless of how well the skill is used (Liu \& Thompson, 2017). People with 
clinical levels of depression report using reappraisal nearly as frequently as those without psychopathology, but consistently report using maladaptive strategies, such as rumination, suppression, or self-blame, more frequently than those without psychopathology (Daros et al., 2018; Sauer et al., 2016). Because we did not measure other emotion regulation strategies in this paradigm, we were unable to test this possibility here, but we encourage researchers to include these measures in future studies.

It should be noted that this moderation result contrasts with Adler et al.'s (2013) findings. In the Adler et al. study, among undergraduates using reappraisal in response to disappointing exam grades, reappraisal quality predicted greater reductions in depressive symptoms among participants with elevated depressive symptoms than those with fewer depressive symptoms. Together, these results may indicate that the interaction between reappraisal quality and depressive symptoms depends on contextual factors. Among people with elevated depressive symptoms, reappraisal quality may be more influential in predicting affective responses to realtime, unexpected situations than recalled memories. Participants in the current study may have had more time to rehearse reappraisals of their memories, which in turn may have developed more consistent affective associations, depending on when the upsetting situation occurred. This may have limited the amount of variability between reappraisal quality and momentary affect during the mood induction, compared to Adler et al.'s design. Further, Adler et al. measured changes in depressive symptoms, whereas we measured changes in affect. Adler et al. reported that changes in positive and negative affect only exhibited small-to-medium sized associations with changes in depressive symptoms. Thus, it is also possible that reappraisal quality exhibits differential effects on affective experiences and depressive symptom changes. We encourage future researchers to explore the contextual factors influencing for whom reappraisal quality 
exerts its effects on various symptomatic and affective experiences.

Researchers have proposed that certain contextual factors (e.g., intensity of negative affect; degree of belief in the malleability of emotions) influence how people with depression choose to use strategies such as reappraisal (Yoon \& Rottenberg, 2019). By adjusting for initial level of negative affect and experimentally induced beliefs in emotion malleability, we were able to statistically test the unique the relations among reappraisal use, reappraisal quality, and affective changes among people with and without clinical levels of depression. We encourage future researchers to test the moderating effects of these contexts in both experimental and naturalistic designs to gain a more comprehensive understanding of reappraisal choice and use.

The results of this study should be considered in light of its limitations. First, given our conceptual model of reappraisal in Hypothesis 2, we modeled reappraisal use as the predictor variable and reappraisal quality as a mediator. However, participants wrote out their reappraisals during the mood induction period before rating the degree to which they used reappraisal. Although we believe this experimental design more accurately captures reappraisal quality by asking participants to write their reappraisals while practicing the skill, we acknowledge that the order in which the tasks were completed does not align with the implied temporal sequence of a mediation model. However, participants' ratings of reappraisal use were completed immediately following the implementation period and were retrospective accounts of the degree of skill use during this period. Further, we have emphasized the associations between reappraisal use and quality and the proportion of the total effect of reappraisal use explained by the indirect effect of reappraisal quality, rather than a causal effect of reappraisal use on quality. It is also possible that participants used their written reappraisals to inform their reappraisal use ratings, inflating the relation between these variables. We attempted to minimize the influence of this design choice 
by using ratings of reappraisal quality from independent coders rather than participants themselves. We relied on overall ratings of reappraisal quality, which are nearly identical $(r=$ .91 ; Strunk et al., 2007) to weighted sums of the quality of individual reappraisal responses. However, because reappraisal quality may be scored using either method (Barber \& DeRubeis, 1992), future researchers might directly compare ratings using each method. Similarly, these are only two ways researchers have used to rate reappraisal quality, and we encourage direct comparisons among multiple methods to better determine the strengths and limitations of each approach. Our analyses were also conducted at the between-person level. Thus, we cannot draw inferences regarding the relation between a given individual using more or less reappraisal and the variable quality of their reappraisals in different situations or at different times. However, future researchers are encouraged to test this hypothesis using more intensive longitudinal designs.

Because participants completed all procedures online, we had less control over their environment and attention than in a laboratory task, although previous researchers have shown that online participants provide data of similar quality to undergraduate and community participants (Necka et al., 2016). We also included multiple attention checks throughout the study and used written responses in our main analyses to reduce the likelihood of distracted or random responses. Finally, although we provided participants with standardized instructions regarding their selection of an autobiographical memory based on previous research, we did not control the amount of time they spent recalling it and it is possible that participants systematically selected memories of varying emotional intensities or with varying degrees of reappraisal affordances (Suri et al., 2018). We limited biases of emotional intensity by controlling for pre-induction affect in our analyses of affective change, although we did not 
require any particular degree of reappraisal affordance. To enhance the reproducibility of this design, we encourage future researchers to standardize the amount of time spent recalling the memory.

In a relatively large sample of online participants, this study provides promising initial evidence that the quality with which people use reappraisal is a mechanism by which this skill contributes to reductions in negative affect and increases in positive affect. We found that this effect was particularly strong among people with fewer depressive symptoms, suggesting that simply using reappraisal to a greater degree may be most effective for people with more depressive symptoms. These findings contribute to a more comprehensive characterization of the process of reappraisal and offer intervention researchers a potential process to target and measure in treatment studies. 


\section{References}

Adler, A. D., Conklin, L. R., \& Strunk, D. R. (2013). Quality of coping skills predicts depressive symptom reactivity over repeated stressors. Journal of Clinical Psychology, 69(12), 1228-1238. https://doi.org/10.1002/jclp.21993

Aldao, A., Nolen-Hoeksema, S., \& Schweizer, S. (2010). Emotion-regulation strategies across psychopathology: A meta-analytic review. Clinical Psychology Review, 30(2), 217-237. https://doi.org/10.1016/j.cpr.2009.11.004

Barber, J. P., \& DeRubeis, R. J. (1992). The Ways of Responding: A scale to assess compensatory skills taught in cognitive therapy. Behavioral Assessment, 14(1), 93-115.

Daros, A. R., Guevara, M. A., Uliaszek, A. A., McMain, S. F., \& Ruocco, A. C. (2018). Cognitive emotion regulation strategies in borderline personality disorder: Diagnostic comparisons and associations with potentially harmful behaviors. Psychopathology, 51(2), 83-95. https://doi.org/10.1159/000487008

Demaree, H. A., Robinson, J. L., Pu, J., \& Allen, J. J. B. (2006). Strategies actually employed during response-focused emotion regulation research: Affective and physiological consequences. Cognition \& Emotion, 20(8), 1248-1260. https://doi.org/10.1080/02699930500405303

Gross, J. J. (1998). The emerging field of emotion regulation: An integrative review. Review of General Psychology, 2(3), 271-299. https://doi.org/10.1037/1089-2680.2.3.271

Gross, J. J., \& John, O. P. (2003). Individual differences in two emotion regulation processes: Implications for affect, relationships, and well-being. Journal of Personality and Social Psychology, 85(2), 348-362. https://doi.org/10.1037/0022-3514.85.2.348

Gruber, J., Hay, A. C., \& Gross, J. J. (2014). Rethinking emotion: Cognitive reappraisal is an 
effective positive and negative emotion regulation strategy in bipolar disorder. Emotion, 14(2), 388-396. https://doi.org/10.1037/a0035249

Hayes, A. F. (2017). Introduction to mediation, moderation, and conditional process analysis: A regression-based approach (2nd ed.). Guilford Press.

Hayes, A. F., \& Krippendorff, K. (2007). Answering the call for a standard reliability measure for coding data. Communication Methods and Measures, 1(1), 77-89. https://doi.org/10.1080/19312450709336664

IBM Corp. (2019). IBM SPSS Statistics for Macintosh, Version 26.0. Armonk, NY: IBM Corp.

Jacobson, N. C., Evey, K. J., Wright, A. G. C., \& Newman, M. G. (2020). Integration of discrete and global structures of affect across three large samples: Specific emotions withinpersons and global affect between-persons. https://doi.org/10.31234/osf.io/gb5up

Katz, B. A., Lustig, N., Assis, Y., \& Yovel, I. (2017). Measuring emotion regulation in the here and now: The development and validation of the State Emotion Regulation Inventory (SERI). Psychological Assessment, 29(10), 1235-1248.

https://doi.org/10.1037/pas0000420

Krieg, E. F. (1999). Biases induced by coarse measurement scales. Educational and Psychological Measurement, 59(5), 749-766. https://doi.org/10.1177/00131649921970125

Krippendorff, K. (2004). Content analysis: An introduction to its methodology (3rd ed.). Sage.

Liu, D. Y., \& Thompson, R. J. (2017). Selection and implementation of emotion regulation strategies in major depressive disorder: An integrative review. Clinical Psychology Review, 57, 183-194. https://doi.org/10.1016/j.cpr.2017.07.004

MacKinnon, D. P., Krull, J. L., Lockwood, C. M. (2000). Equivalence of the mediation, 
confounding and suppression effect. Prevention Science, 1(4), 173-181. https://doi.org/10.1023/a:1026595011371

McRae, K., Ciesielski, B., \& Gross, J. J. (2012). Unpacking cognitive reappraisal: Goals, tactics, and outcomes. Emotion, 12(2), 250-255. https://doi.org/10.1037/a0026351

Millgram, Y., Joormann, J., Huppert, J. D., \& Tamir, M. (2015). Sad as a matter of choice? Emotion-regulation goals in depression. Psychological Science, 26(8), 1216-1228. https://doi.org/10.1177/0956797615583295

Necka, E. A., Cacioppo, S., Norman, G. J., \& Cacioppo, J. T. (2016). Measuring the prevalence of problematic respondent behaviors among MTurk, campus, and community participants. PLoS ONE, 11(6), e0157732. https://doi.org/10.1371/journal.pone.0157732

Olatunji, B. O., Berg, H. E., \& Zhao, Z. (2017). Emotion regulation of fear and disgust: differential effects of reappraisal and suppression. Cognition \& Emotion, 31(2), 403-410. https://doi.org/10.1080/02699931.2015.1110117

Pasupathi, M., Wainryb, C., Mansfield, C. D., \& Bourne, S. (2017). The feeling of the story: Narrating to regulate anger and sadness. Cognition \& Emotion, 31(3), 444-461. https://doi.org/10.1080/02699931.2015.1127214

Quigley, L., \& Dobson, K. S. (2014). An examination of trait, spontaneous and instructed emotion regulation in dysphoria. Cognition \& Emotion, 28(4), 622-635. https://doi.org/10.1080/02699931.2013.848786

Radloff, L. S. (1977). The CES-D Scale: A self-report depression scale for research in the general population. Applied Psychological Measurement, 1(3), 385-401. https://doi.org/10.1177/014662167700100306

Rowlands, L., Coetzer, R., \& Turnbull, O. (2020). This time it's personal: Reappraisal after 
acquired brain injury. Cognition \& Emotion, 35(2), 305-323.

\section{https://doi.org/10.1080/02699931.2020.1839384}

Rusting, C. L., \& Nolen-Hoeksema, S. (1998). Regulating responses to anger: Effects of rumination and distraction on angry mood. Journal of Personality and Social Psychology, 74(3), 790-803. https://doi.org/10.1037/0022-3514.74.3.790

Sauer, C., Sheppes, G., Lackner, H. K., Arens, E. A., Tarrasch, R., \& Barnow, S. (2016). Emotion regulation choice in female patients with borderline personality disorder: Findings from self-reports and experimental measures. Psychiatry Research, 242, 375384. https://doi.org/10.1016/j.psychres.2016.04.113

Smoski, M. J., LaBar, K. S., \& Steffens, D. C. (2014). Relative effectiveness of reappraisal and distraction in regulation emotion in late-life depression. The American Journal of Geriatric Psychiatry, 22(9), 898-907. https://doi.org/10.1016/j.jagp.2013.01.070

Song, Y., Jordan, J. I., Shaffer, K. A., Wing, E. K., McRae, K., \& Waugh, C. (2019). Effects of incidental positive emotion and cognitive reappraisal on affective responses to negative stimuli. Cognition \& Emotion, 33(6), 1155-1168. https://doi.org/10.1080/02699931.2018.1541789

Southward, M. W., \& Cheavens, J. S. (2020). Quality or quantity? A multistudy analysis of emotion regulation skills deficits associated with borderline personality disorder. Personality Disorders: Theory, Research, \& Treatment, 11(1), 24-35.

\section{https://doi.org/10.1037/per0000357}

Southward, M. W., Heiy, J. E., \& Cheavens, J. S. (2019). Emotions as context: Do the naturalistic effects of emotion regulation strategies depend on the regulated emotion? Journal of Social \& Clinical Psychology, 38(6), 451-474. 
https://doi.org/10.1521/jscp.2019.38.6.451

Southward, M. W., Sauer-Zavala, S., \& Cheavens, J. S. (2021). Specifying the mechanisms and targets of emotion regulation: A translational framework from affective science to psychological treatment. Clinical Psychology: Science and Practice. Advance online publication. https://doi.org/10.1037/cps0000003

Strunk, D. R., Adler, A. D., \& Hollars, S. N. (2013). Cognitive therapy skills predict cognitive reactivity to sad mood following cognitive therapy for depression. Cognitive Therapy and Research, 37(6), 1214-1219. https://doi.org/10.1007/s10608-013-9570-z

Strunk, D. R., DeRubeis, R. J., Chiu, A. W., \& Alvarez, J. (2007). Patients' competence in and performance of cognitive therapy skills: Relation to the reduction of relapse risk following treatment for depression. Journal of Consulting and Clinical Psychology, 75(4), 523-530. https://doi.org/10.1037/0022-006X.75.4.523

Suri, G., Sheppes, G., Young, G., Abraham, D., McRae, K., \& Gross, J. J. (2018). Emotion regulation choice: The role of environmental affordances. Cognition \& Emotion, 32(5), 963-971. https://doi.org/10.1080/02699931.2017.1371003

Tamir, M., Halperin, E., Porat, R., Bigman, Y. E., \& Hasson, Y. (2019). When there's a will, there's a way: Disentangling the effects of goals and means in emotion regulation. Journal of Personality and Social Psychology, 116(5), 795-816. https://doi.org/10.1037/pspp0000232

Vilagut, G., Forero, C. G., Barbaglia, G., \& Alonso, J. (2016). Screening for depression in the general population with the Center for Epidemiologic Studies Depression (CES-D): A systematic review with meta-analysis. PloS ONE, 11(5), e0155431.

https://doi.org/10.1371/journal.pone.0155431 
Vishkin, A., Hasson, Y., Millgram, Y., \& Tamir, M. (2019). One size does not fit all: Tailoring cognitive reappraisal to different emotions. Personality and Social Psychology Bulletin, 46(3), 469-484. https://doi.org/10.1177/0146167219861432

Watson, D., \& Stanton, K. (2017). Emotion blends and mixed emotions in the hierarchical structure of affect. Emotion Review, 9(2), 99-104. https://doi.org/10.1177/1754073916639659

Webb, T. L., Miles, E., \& Sheeran, P. (2012). Dealing with feeling: A meta-analysis of the effectiveness of strategies derived from the process model of emotion regulation. Psychological Bulletin, 138(4), 775-808. https://doi.org/10.1037/a0027600

Weber, H., Loureiro de Assunção, V., Martin, C., Westmeyer, H., \& Geisler, F. C. (2014). Reappraisal inventiveness: The ability to create different reappraisals of critical situations. Cognition \& Emotion, 28(2), 345-360. https://doi.org/10.1080/02699931.2013.832152

Wu, X. F., Guo, T. T., Tang, T. T., Shi, B. G., \& Luo, J. (2017). Role of creativity in the effectiveness of cognitive reappraisal. Frontiers in Psychology, 8, 1598. https://doi.org/10.3389/fpsyg.2017.01598

Wu, X. F., Guo, T. T., Tan, T. T., Zhang, W. C., Qin, S. Z., Fan, J., \& Luo, J. (2019). Superior emotional regulating effects of creative cognitive reappraisal. Neuroimage, 200, 540551. https://doi.org/10.1016/j.neuroimage.2019.06.061

Yin, S., Gibbons, M. B. C., Diehl, C., Gallop, R., \& Crits-Christoph, P. (2018). A self-report version of the Ways of Responding: Reliability and validity in a clinical sample. Psychotherapy Research, 28(4), 581-592. https://doi.org/10.1080/10503307.2016.1233367 
Yoon, S., \& Rottenberg, J. (2019). Why do people with depression use faulty emotion regulation strategies? Emotion Review, 12(2), 118-128. https://doi.org/10.1177/1754073919890670

Yzerbyt, V., Muller, D., Batailler, C., \& Judd, C. M. (2018). New recommendations for testing indirect effects in mediational models: The need to report and test component paths. Journal of Personality and Social Psychology, 115(6), 929-943.

https://doi.org/10.1037/pspa0000132

Zeier, P., Sandner, M., \& Wessa, M. (2020). Script-based reappraisal test introducing a new paradigm to investigate the effect of reappraisal inventiveness on reappraisal effectiveness. Cognition \& Emotion, 34(4), 793-799.

https://doi.org/10.1080/02699931.2019.1663153 
Table 1

Correlations Among Demographics and Measures of Interest

\begin{tabular}{|c|c|c|c|c|c|c|c|c|c|c|c|c|c|}
\hline Measures of Interest & 1 & 2 & 3 & 4 & 5 & 6 & 7 & 8 & 9 & 10 & 11 & 12 & 13 \\
\hline \multicolumn{14}{|l|}{ 1. Age } \\
\hline 2. Gender & $-.21 * *$ & & & & & & & & & & & & \\
\hline 3. Racial Background & $-.20 * *$ & .03 & & & & & & & & & & & \\
\hline 4. Education & $.15^{* *}$ & $-.14 *$ & .08 & & & & & & & & & & \\
\hline 5. SERI-R & .06 & -.03 & -.02 & .08 & & & & & & & & & \\
\hline 6. Reappraisal Quality & .06 & .05 & $-.12 *$ & .07 & $.56 * *$ & & & & & & & & \\
\hline 7. Word Count & .06 & -.11 & $-.19^{*}$ & $.14^{*}$ & .04 & .09 & & & & & & & \\
\hline 8. ERQ-R & .10 & -.06 & .04 & .01 & $.14^{*}$ & -.01 & .08 & & & & & & \\
\hline 9. NA Post-Induction & -.08 & -.08 & $<.01$ & -.02 & $-.13^{*}$ & $-.18^{* *}$ & -.05 & $-.12 *$ & & & & & \\
\hline 10. NA Post-Implementation & -.07 & -.09 & -.02 & -.01 & $-.38 * *$ & $-.42 * *$ & .03 & -.07 & $.71 * *$ & & & & \\
\hline 11. PA Post-Induction & .03 & .08 & -.09 & .05 & $.20 * *$ & .11 & .02 & $.29 * *$ & $-.43 * *$ & $-.32 * *$ & & & \\
\hline 12. PA Post-Implementation & .03 & .06 & $<-.01$ & .06 & $.43^{* *}$ & $.39 * *$ & -.10 & $.20 * *$ & $-.32 * *$ & $-.52 * *$ & $.72 * *$ & & \\
\hline 13. CES-D & $-.16^{* *}$ & .05 & .06 & -.08 & -.06 & -.09 & -.06 & $-.30 * *$ & $.42 * *$ & $.35^{* *}$ & $-.38 * *$ & $-.35 * *$ & \\
\hline$M / n$ & 36.30 & 160 & 218 & $\dagger$ & 5.00 & 2.11 & 125.43 & 5.25 & 20.40 & 21.55 & 41.87 & 41.48 & 13.97 \\
\hline$S D / \%$ & 11.39 & 51.0 & 69.4 & & 1.53 & 1.15 & 50.22 & 1.05 & 20.18 & 21.68 & 29.67 & 31.89 & 11.74 \\
\hline
\end{tabular}

Note. Gender dichotomized: female $=0$, male $=1$. Racial Background dichotomized: White $=0$, Non-White $=1$. All statistics with Education are Spearman's $\rho$ because participants indicated specific educational attainment, rather than years in school. SERI-R $=$ State Emotion Regulation Inventory - Reappraisal subscale. ERQ-R $=$ Emotion Regulation Questionnaire - Reappraisal subscale. NA = Negative Affect. Negative Affect is a mean composite of disgust, fear, and sadness. PA $=$ Positive Affect. CES-D = Center for Epidemiologic Studies - Depression scale.

${ }^{\dagger}$ The median level of education completed was a 2 -year associate degree.

$* p<.05, * * p<.01$ 
Table 2

Conditional Direct and Indirect Effects of Reappraisal Use and Quality on Changes in Negative and Positive Affect by Depressive Symptom Severity

Changes in Negative Affect

Conditional Direct Effect of Reappraisal Use

\begin{tabular}{|c|c|c|c|c|}
\hline \multicolumn{2}{|c|}{ CES-D } & $B$ & $S E$ & $95 \% \mathrm{CI}$ \\
\hline$-1 S D$ & 2.16 & -2.77 & .82 & {$[-4.37,-1.16]$} \\
\hline $0 S D$ & 13.85 & -2.88 & .60 & {$[-4.07,-1.69]$} \\
\hline$+1 S D$ & 25.53 & -3.00 & .88 & {$[-4.73,-1.26]$} \\
\hline
\end{tabular}

Changes in Positive Affect

Conditional Indirect Effect of Reappraisal

Conditional Direct Effect of Reappraisal Use

\begin{tabular}{|c|c|c|c|c|}
\hline \multicolumn{2}{|c|}{ CES-D } & $B$ & $S E$ & $95 \% \mathrm{CI}$ \\
\hline$-1 S D$ & 2.16 & 3.14 & 1.17 & {$[.84,5.44]$} \\
\hline $0 S D$ & 13.85 & 3.78 & .87 & {$[2.07,5.49]$} \\
\hline$+1 S D$ & 25.53 & 4.42 & 1.26 & {$[1.94,6.91]$} \\
\hline
\end{tabular}

Note. CES-D = Center for Epidemiologic Studies - Depression scale. Standard errors and confidence intervals for conditional indirect effects based on 10,000 percentile bootstraps. Quality

\begin{tabular}{|c|c|c|c|c|}
\hline \multicolumn{2}{|c|}{ CES-D } & $B$ & $S E$ & $95 \% \mathrm{CI}$ \\
\hline$-1 S D$ & 2.16 & -2.16 & .45 & {$[-3.11,-1.34]$} \\
\hline $0 S D$ & 13.85 & -1.38 & .32 & {$[-2.08,-.80]$} \\
\hline$+1 S D$ & 25.53 & -.63 & .50 & {$[-1.70, .27]$} \\
\hline
\end{tabular}
Quality

\begin{tabular}{rrlcc}
\hline \multicolumn{2}{c}{ CES-D } & \multicolumn{1}{c}{$B$} & \multicolumn{1}{c}{$S E$} & $95 \%$ CI \\
\hline$-1 S D$ & 2.16 & 3.67 & .76 & {$[2.30,5.31]$} \\
$0 S D$ & 13.85 & 2.50 & .54 & {$[1.50,3.64]$} \\
$+1 S D$ & 25.53 & 1.37 & .73 & {$[.04,2.92]$} \\
\hline
\end{tabular}




\section{Figure 1}

Study Flow and Procedures

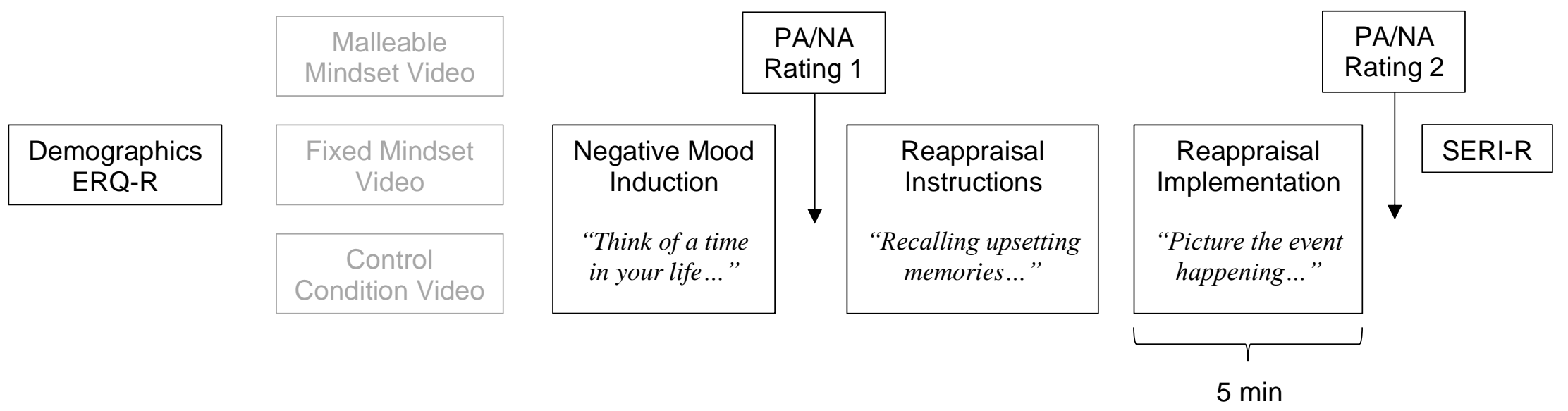

$E P Q-R=$ Emotion Regulation Questionnaire-Reappraisal subscale. $\mathrm{PA}=$ Positive Affect. NA = Negative Affect. SERI-R = State Emotion Regulation Inventory - Reappraisal subscale. Black boxes indicate measures or procedures directly involved in the current study. Grey boxes indicate measures or procedures conducted as part of a larger parent study with the potential to impact the current study. 


\section{Figure 2}

The Indirect Effect of Reappraisal Use on Changes in Negative and Positive Affect Through Reappraisal Quality

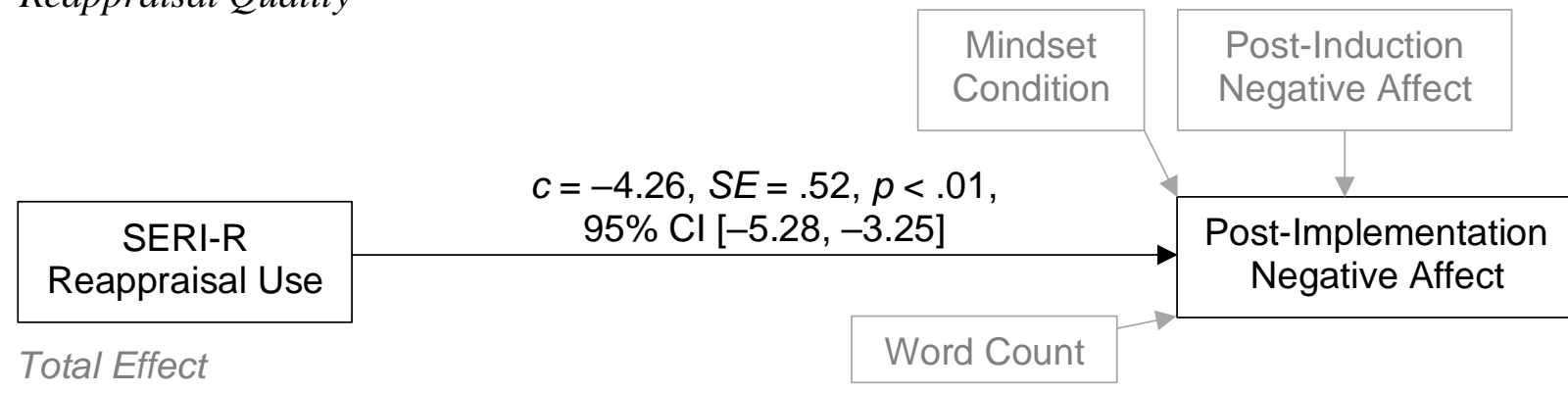

\section{Direct \& Indirect Effects}

$a b=-1.49, S E=.33$,

$95 \% \mathrm{Cl}[-2.17,-.90]$

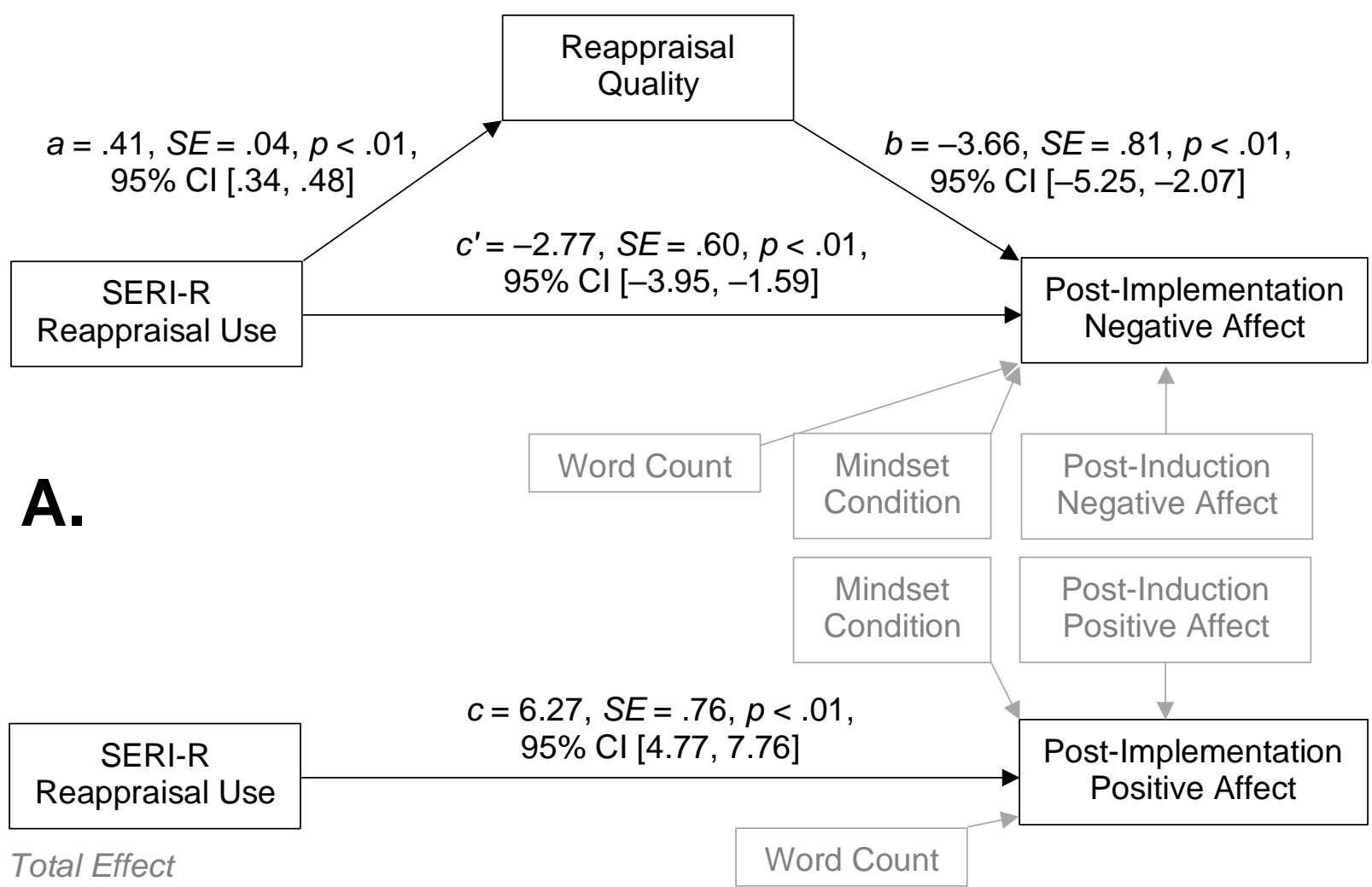

Direct \& Indirect Effects

$a b=2.67, S E=.54$,

$95 \% \mathrm{Cl}[1.64,3.79]$

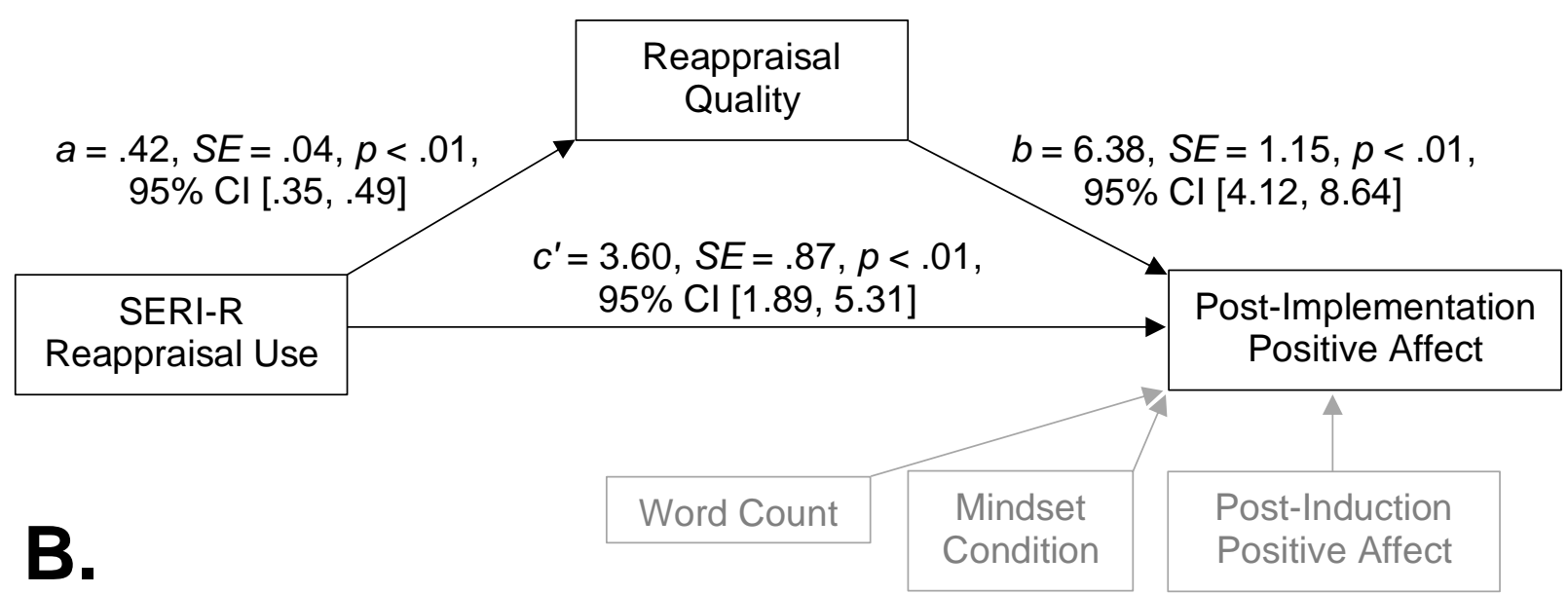




\section{Figure 3}

Depressive Symptoms Moderate the Relations Between Reappraisal Quality and Changes in Negative and Positive Affect From Post-Mood Induction to Post-Reappraisal Implementation

15

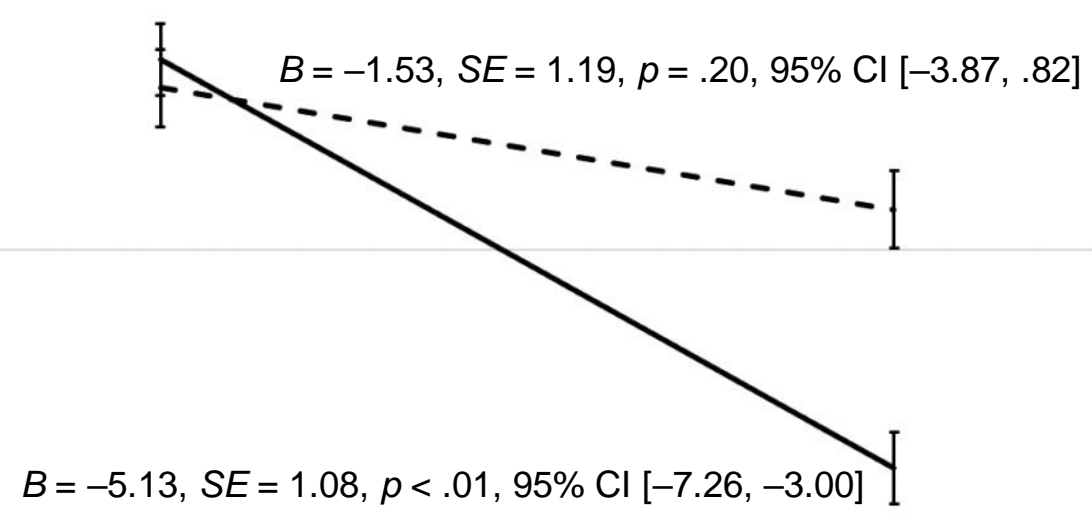

A. ${ }^{-10}$

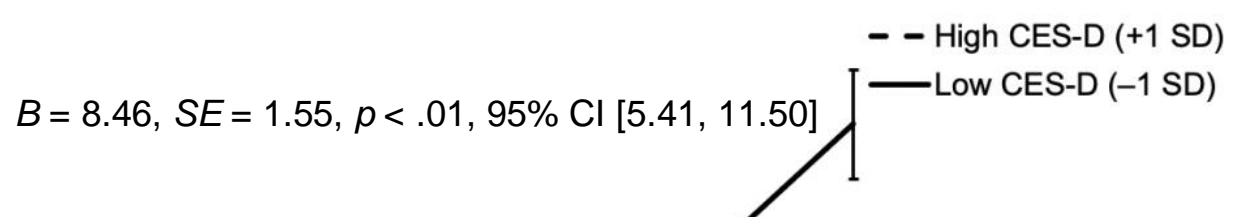

10

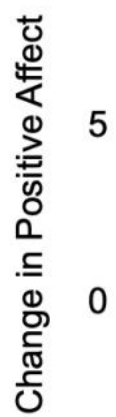

$-5$

B. $^{-10}$

Low Quality (-1 SD)

High Quality (+1 SD) 